\title{
A new time domain approach to evaluate transmission loss in layered partitions
}

\author{
Roberto A. Tenenbaum, Marcelo Bruno S. Magalhães, Moysés Zindeluk \\ Acoustics and Vibration Laboratory, Federal University of Rio de Janeiro, C.P. 68503, 21945-970, Rio de Janeiro, Brazil
}

\begin{abstract}
The insulation provided by layered barriers is addressed from a theoretical point of view. An exact time-domain solution for the normal incidence plane wave propagation in layered media excited by an arbitrary input is presented, leading to a fast algorithm to compute the transmission loss in a compound wall. Numerical experiments demonstrate its agreement with the mass law for a single wall. For multiple partitions, however, the mass law is seen to be inadequate to predict correctly the transmission loss and deviates from the model presented when the number of layers increases. It is also shown that, by changing the material of the internal layers, it is possible to achieve a better insulation than that obtained by using a single wall with a greater density and the same thickness. The theoretical model presented here provides an easy way to evaluate the transmission loss and leads to a procedure for the determination of the optimal choice of materials to be used in a partition.
\end{abstract}

\section{INTRODUCTION}

The main parameter in determining the acoustic insulation capability of a partition is its acoustic impedance. Despite the existence of other phenomena such as flexural waves, damping and resonances, the principal property of a barrier which generates wave rejection is the impedance jump introduced by the presence of two different materials in the sound propagation path. When very efficient insulation is required, specially when there is not enough space or when the overall mass is critical (e.g., in aerospace applications), improved performance can be achieved by using an adequately-designed, layered partition. It is required, however, to use a new design criterion, that is capable of estimating with good accuracy the transmission loss that will be produced. It is well known that a double wall works better than a single one with the same overall mass, but when the number of layers is increased, the theoretical evaluation of the transmitted intensity level becomes more and more involved. This paper presents a method for this evaluation in the particular case when the medium is excited by plane waves. Although we will consider here only one-dimensional wave propagation in lossless elastic media, conservative results are expected, since losses improve the insulation.

The scattering of acoustic waves by inhomogeneous one-dimensional media has been considered in many fields of practical interest. Approximate methods can furnish rough evaluations of the wave behaviour. The classical WKB was adopted by Hassab, ${ }^{1}$ the Born approximation was applied by Levine, ${ }^{2}$ and Candel et al $^{3}$ proposed the forward-scattering approximation, in the frequency domain. Tenenbaum and Zindeluk ${ }^{4}$ proposed an approximate method in the time domain, arriving at an exact inversion equation for some particular wavelets.

For strong inhomogeneity, all these techniques fail and the method of characteristics has been shown to be very useful. Most of the important applications concern the inverse reflection problem and very interesting surveys on the subject are found in Schwetlick ${ }^{\mathbf{5}}$ and Mendel and Ashrafi. ${ }^{6}$ The Z-transform approach is very common, follow- ing the pioneering work of Ware and $\mathrm{Aki},{ }^{7}$ which applies the Gelfand-Levitan theory to solve Schroedinger's equation.

The transmission response problem has attracted the interest of many researchers. Claerbout ${ }^{8}$ proposed a method to obtain the synthesis of a layered medium from its transmission response. More recently Carrol and Santosa ${ }^{9}$ and Yagle $^{10}$ presented fast algorithms to reconstruct the impedance profile from the impulse transmission response of the medium. These methods relate the transmission response to the reflection response and try to recover the impedance profile based on the latter. As far as we know, there is no exact solution for the transmitted impulse response of a lossless layered medium and, moreover, no exact equation for the transmitted response of such a medium subjected to an arbitrary input.

In this paper, starting from the characteristic variables formulation, a compact form of the wave equation is achieved and a general algebraic solution for the transmission response is found for layered media subjected to an arbitrary input.

\section{THEORETICAL BACKGROUND}

The problem of plane wave propagation in a one-dimensional, inhomogeneous, transversely-infinite, linear elastic medium is depicted in Fig. 1. Regions $x<0$ and $x>L$ are homogenous, while in the interval $0 \leq x \leq L$ there is a inhomogenous inclusion. The mechanical properties $\rho(x)$, $E(x)$ and $c(x)=[E(x) / \rho(x)]^{1 / 2}$, respectively the density, the effective elastic modulus and the longitudinal wave speed are functions of $x$, the spatial coordinate. The medium is subjected to the stress wave $f(t)$ at $x=0$ and, as a result of its inhomogeneity, reflected and transmitted waves are generated, respectively, $g(t)$ and $h(t)$.

The constitutive equation is

$$
\sigma(x, t)=E(x) u_{x}(x, t)
$$

where $\sigma(x, t)$ is the longitudinal stress function of space and time, $u(x, t)$ is the longitudinal displacement, and the index $x$ denotes partial differentiation with respect to $x$. 